\title{
SERIES IN LE ROY TYPE FUNCTIONS: INEQUALITIES AND CONVERGENCE THEOREMS
}

\author{
Jordanka Paneva-Konovska \\ Institute of Mathematics and Informatics \\ Bulgarian Academy of Sciences \\ 'Acad. G. Bontchev' Street, Block 8 \\ Sofia-1113, BULGARIA
}

\begin{abstract}
In studying the behaviour of series defined by means of the Le Roy type functions, we prove Cauchy-Hadamard type theorems. Asymptotic formulae for these new special functions in the cases of 'large' values of indices are also provided, as necessary in the proofs of the convergence theorems for the considered series in the complex plane.
\end{abstract}

AMS Subject Classification: 30D20, 33E12, 30A10, 30D15, 40A30

Key Words: Le Roy functions; inequalities; asymptotic formula; CauchyHadamard theorem

\section{Introduction}

In two recent papers, Gerhold [7] and independently Garra and Polito [5], introduced the new special function

$$
F_{\alpha, \beta}^{(\gamma)}(z)=\sum_{k=0}^{\infty} \frac{z^{k}}{[\Gamma(\alpha k+\beta)]^{\gamma}}, \quad z \in \mathbb{C}, \alpha, \beta, \gamma \in \mathbb{C},
$$

which is an entire function of the complex variable $z$ for all values of the parameters such that

$$
\Re(\alpha)>0, \beta \in \mathbb{C}, \gamma>0 .
$$

In fact, the function (1) is introduced for the values of parameters $\alpha>0, \beta>$

Received: September 25, 2020

(C) 2020 Academic Publications 
$0, \gamma>0$, and on a later stage its definition is extended to the range (2) by Garrappa, Rogosin and Mainardi [6].

The function $F_{\alpha, \beta}^{(\gamma)}$ is closely related to the classical modified Bessel function of the first kind $I_{0}(2 \sqrt{z})$,

$$
I_{0}(2 \sqrt{z})=\sum_{k=0}^{\infty} \frac{z^{k}}{(k !)^{2}}, \quad z \in \mathbb{C}
$$

as well as to the 2-parametric Mittag-Leffler function (see for example, the recent monographs [8] and [23]),

$$
E_{\alpha, \beta}(z)=\sum_{k=0}^{\infty} \frac{z^{k}}{\Gamma(\alpha k+\beta)}, \quad z \in \mathbb{C} ; \Re(\alpha)>0, \beta \in \mathbb{C} .
$$

The Mittag-Leffler functions (4) have been studied in details by Dzrbashjan [3], [4]: asymptotic formulae in different parts of the complex plane, distribution of the zeros, kernel functions of inverse Borel type integral transforms, various relations and representations. The detailed properties of these functions can be found in the monographs of Kilbas et al. [11], Podlubny [27], Gorenflo et al. [8], etc. The function (4) was named after the great Swedish mathematician Gösta Magnus Mittag-Leffler (1846-1927) who defined it in the 1-parameter case $\left(E_{\alpha}(z)\right.$ with $\left.\beta=1\right)$ by a power series and studied its properties in $1902-$ 1905 (see detailed description in [8]). As a matter of fact, Mittag-Leffler introduced the function $E_{\alpha}(z)$ for the purposes of his method for summation of divergent series. Later, the function (4) was recognized as the 'Queen function of fractional calculus' [9, 18, 29], see also [23], for its basic role for analytic solutions of fractional order integral and differential equations and systems. In the recent decades, successful applications of the Mittag-Leffler function and its generalizations in problems of physics, biology, chemistry, engineering and other applied sciences made it better known among scientists. A considerable literature is devoted to the investigation of the analyticity properties of this function; among the references of [8] and [23] there are quoted several authors who, after Mittag-Leffler, have investigated such a function from a pure mathematical, application oriented and numerical point of view.

The function $F_{\alpha, \beta}^{(\gamma)}$ is also closely related to the multi-index extensions of (4) (with $2 m$ and $3 m$ parameters, $m=1,2, \ldots$, see $[1,12,14,10,23]$ ), i.e. the so-called multi-index Mittag-Leffler functions. The $(2 \mathrm{~m})$ multi-index MittagLeffler function was introduced in Yakubovich and Luchko [34] and studied in details by Kiryakova [12, 13], defined by the formula 


$$
E_{\left(\alpha_{i}\right),\left(\beta_{i}\right)}(z)=E_{\left(\alpha_{i}\right),\left(\beta_{i}\right)}^{m}(z)=\sum_{k=0}^{\infty} \frac{z^{k}}{\Gamma\left(\alpha_{1} k+\beta_{1}\right) \ldots \Gamma\left(\alpha_{m} k+\beta_{m}\right)},
$$

for $z \in \mathbb{C}$ and $m>1$. The parameters $\alpha_{i}, \beta_{i}$ are all complex for $i=1,2, \ldots m$ and $\Re\left(\alpha_{i}\right)>0$. The next extension, with $(3 m)$ indices, has additional $m$ complex parameters $\gamma_{i}$. It was introduced and studied in details by PanevaKonovska (for its properties see e. g. [23]), also as an extension of the 3parameter Mittag-Leffler type function, known as the Prabhakar function. It is defined by the formula

$$
E_{\left(\alpha_{i}\right),\left(\beta_{i}\right)}^{\left(\gamma_{i}\right), m}(z)=\sum_{k=0}^{\infty} \frac{\left(\gamma_{1}\right)_{k} \ldots\left(\gamma_{m}\right)_{k}}{\Gamma\left(\alpha_{1} k+\beta_{1}\right) \ldots \Gamma\left(\alpha_{m} k+\beta_{m}\right)} \frac{z^{k}}{(k !)^{m}},
$$

where $(\gamma)_{k}$ is the Pochhammer symbol: $(\gamma)_{k}=\gamma(\gamma+1) \ldots(\gamma+k-1), k=$ $1,2, \ldots,(\gamma)_{0}=1$.

Actually if the parameter $\gamma=m$ is a positive integer, the function $F_{\alpha, \beta}^{(\gamma)}$ can be presented as a multi-index Mittag-Leffler function (5) with all equal $\alpha$ 's and $\beta$ 's.

The function (1) is a natural extension of the Le Roy function

$$
F^{(\gamma)}(z)=F_{1,1}^{(\gamma)}(z)=\sum_{k=0}^{\infty} \frac{z^{k}}{[k !]^{\gamma}}=\sum_{k=0}^{\infty} \frac{z^{k}}{[\Gamma(k+1)]^{\gamma}}, \quad z \in \mathbb{C}, \gamma \in \mathbb{C},
$$

named after the French mathematician Édouard Louis Emmanuel Julien Le Roy (1870-1954). Le Roy himself introduced it in [17] for the purposes to study the asymptotics of the analytic continuation of the sum of power series. This reason for the origin of (7) sounds close to the Mittag-Leffler's idea to introduce the function $E_{\alpha}(z)$ for the aims of analytic continuation, and it seems that MittagLeffler and Le Roy were working on this idea in competition. The Le Roy function is involved in the solution of problems of various types; in particular it has been recently used in the construction of a Conway-Maxwell-Poisson distribution [2] which is important due to its ability to model count data with different degrees of over- and under-dispersion [28, 30].

For the sake of brevity, in this paper we often use the name Le Roy type function for the Le Roy type function $F_{\alpha, \beta}^{(\gamma)}$ defined by (1).

The study of the asymptotic behavior of the Le Roy type function is of special interest due to the existing and perspective applications. Thus, the work of Gerhold [7] was devoted to study the asymptotic properties of $F_{\alpha, \beta}^{(\gamma)}(z)$ as an entire function in some sectors of the complex plane. It was shown by 
Garrappa, Rogosin and Mainardi [6, Lemma 2.1] that this function has order $\rho$ and type $\sigma$, as follows

$$
\rho=\frac{1}{\gamma \Re(\alpha)}, \quad \sigma=\gamma,
$$

if the parameters satisfy the conditions (2).

Let us note that the above results conform with the corresponding ones for the order and type of the Mittag-Leffler function (4) as well as of its multi-index extensions (5) and (6) (see details in [12, 15, 10, 8] and [23], as well).

In the series of papers [19]-[22], [24]-[26], as well as in the recent book [23], we studied series in systems of some special functions of fractional calculus, as for example fractional indices analogues of the Bessel functions and multi-index Mittag-Leffler functions and we proved various results on their convergence in the complex domain.

In this paper, devoted to the Le Roy type functions (1), we prove for them some inequalities in the complex plane $\mathbb{C}$ and on its compact subsets, asymptotic formulae for 'large' values of their indices of and find the domain of convergence of series in such kind of functions.

\section{Inequalities and asymptotic formula}

For our purposes we consider the family of functions

$$
F_{\alpha, n}^{(\gamma)}(z)=\sum_{k=0}^{\infty} \frac{z^{k}}{[\Gamma(\alpha k+n)]^{\gamma}}, \quad z \in \mathbb{C} ; n \in \mathbb{N}_{0}, \alpha>0, \gamma>0,
$$

where $\mathbb{N}_{0}$ means the set of nonnegative integers, i.e. $\mathbb{N}_{0}=\mathbb{N} \cup\{0\}$.

We deal with some analytical transformations of the function (9) for each value of the parameter $n$. The following result can be formulated.

Lemma 1. Let $z \in \mathbb{C}, \alpha>0, \gamma>0$ and $n \in \mathbb{N}_{0}$. Then there exists a number $p \in \mathbb{N}_{0}$ and an entire function $\vartheta_{\alpha, n}^{(\gamma)}$ such that

$$
F_{\alpha, n}^{(\gamma)}(z)=\frac{z^{p}}{[\Gamma(\alpha p+n)]^{\gamma}}\left(1+\vartheta_{\alpha, n}^{(\gamma)}(z)\right),
$$

with the following value of $p$ :

(I) $p=0$ for $n \in \mathbb{N}$,

(II) $p=1$ for $n=0$. 
Proof. The cases $n \in \mathbb{N}$ and $n=0$ are considered separately. Beginning with $n \in \mathbb{N}$, we note that in this case $\alpha k+n>0$ for all the values of $k=0,1, \ldots$ Therefore all the coefficients in the function (9) are different from 0 . Then this function is presented in the form

with

$$
F_{\alpha, n}^{(\gamma)}(z)=\frac{1}{[\Gamma(n)]^{\gamma}}\left(1+\vartheta_{\alpha, n}^{(\gamma)}(z)\right)
$$

$$
\vartheta_{\alpha, n}^{(\gamma)}(z)=[\Gamma(n)]^{\gamma} \sum_{k=1}^{\infty} \frac{z^{k}}{[\Gamma(\alpha k+n)]^{\gamma}}
$$

Let now $n=0$. In this case the function (9) is reduced to

$$
F_{\alpha, 0}^{(\gamma)}(z)=\sum_{k=1}^{\infty} \frac{z^{k}}{[\Gamma(\alpha k)]^{\gamma}}=\frac{z}{[\Gamma(\alpha)]^{\gamma}}\left(1+\vartheta_{\alpha, 0}^{(\gamma)}(z)\right)
$$

with

$$
\vartheta_{\alpha, 0}^{(\gamma)}(z)=[\Gamma(\alpha)]^{\gamma} \sum_{k=2}^{\infty} \frac{z^{k-1}}{[\Gamma(\alpha k)]^{\gamma}}=[\Gamma(\alpha)]^{\gamma} \sum_{k=1}^{\infty} \frac{z^{k}}{[\Gamma(\alpha(k+1))]^{\gamma}}
$$

Let us note that (12) and (14) are entire functions. This property follows from the identities (11) and (13) and from the holomorphicity of $F_{\alpha, n}^{(\gamma)}(z)$ in the whole complex plane. Further, the formula (10) follows in view of (11) and (13) with the corresponding $p$.

Remark 2. Note that the functions (12) and (14) can be written together as follows

$$
\vartheta_{\alpha, n}^{(\gamma)}(z)=[\Gamma(\alpha p+n)]^{\gamma} \sum_{k=p+1}^{\infty} \frac{z^{k-p}}{[\Gamma(\alpha k+n)]^{\gamma}}, \quad n \in \mathbb{N}_{0},
$$

with the corresponding values of $p$, namely:

$$
\begin{aligned}
& \text { (I) } p=0 \text { for } n \in \mathbb{N}, \\
& \text { (II) } p=1 \text { for } n=0 .
\end{aligned}
$$

Lemma 3. Let $z \in \mathbb{C}, \alpha>0, \gamma>0$ and $n \in \mathbb{N}$ and let $K \subset \mathbb{C}$ be a nonempty compact set. Then the entire function $\vartheta_{\alpha, n}^{\gamma}$, given by (12), satisfies the following inequality

$$
\left|\vartheta_{\alpha, n}^{(\gamma)}(z)\right| \leq \frac{[\Gamma(\alpha+1)]^{\gamma}[\Gamma(n)]^{\gamma}}{[\Gamma(\alpha+n)]^{\gamma}}\left(F_{\alpha, 1}^{(\gamma)}(|z|)-1\right), \quad z \in \mathbb{C},
$$


and moreover there exists a constant $C=C(K), 0<C<\infty$, such that

$$
\left|\vartheta_{\alpha, n}^{(\gamma)}(z)\right| \leq C \frac{[\Gamma(n)]^{\gamma}}{[\Gamma(\alpha+n)]^{\gamma}}
$$

for all the natural numbers $n$ and each $z \in K$.

Proof. Note that the function (12) can be rewritten in the following equivalent form

$$
\vartheta_{\alpha, n}^{(\gamma)}(z)=\frac{[\Gamma(n)]^{\gamma}}{[\Gamma(\alpha+n)]^{\gamma}} \sum_{k=1}^{\infty} \frac{[\Gamma(\alpha+n)]^{\gamma}}{[\Gamma(\alpha k+n)]^{\gamma}} z^{k}
$$

and let us denote

$$
\widetilde{\gamma}_{n, k}=\frac{[\Gamma(\alpha+n)]^{\gamma}}{[\Gamma(\alpha k+n)]^{\gamma}}, \quad \widetilde{u}_{n, k}(z)=\widetilde{\gamma}_{n, k} z^{k} .
$$

Then we consecutively obtain the following estimations for (19):

$$
\widetilde{\gamma}_{n, 1}=1
$$

and for $k \in \mathbb{N}, k \neq 1$ :

$$
\begin{gathered}
0<\widetilde{\gamma}_{n, k}=\frac{[\Gamma(\alpha+1)]^{\gamma}}{[\Gamma(\alpha k+1)]^{\gamma}} \prod_{s=1}^{n-1} \frac{(\alpha+s)^{\gamma}}{(\alpha k+s)^{\gamma}} \leq \frac{[\Gamma(\alpha+1)]^{\gamma}}{[\Gamma(\alpha k+1)]^{\gamma}}, \\
\left|\widetilde{u}_{n, k}(z)\right|=\widetilde{\gamma}_{n, k}|z|^{k} \leq \frac{[\Gamma(\alpha+1)]^{\gamma}}{[\Gamma(\alpha k+1)]^{\gamma}}|z|^{k}, \text { for } k \in \mathbb{N} .
\end{gathered}
$$

Therefore,

$$
\left|\vartheta_{\alpha, n}(z)\right| \leq \frac{[\Gamma(n)]^{\gamma}[\Gamma(\alpha+1)]^{\gamma}}{[\Gamma(\alpha+n)]^{\gamma}}\left(\sum_{k=0}^{\infty} \frac{|z|^{k}}{[\Gamma(\alpha k+1)]^{\gamma}}-1\right),
$$

which proves (16) in the whole complex plane.

Further, for all $z$ on the compact set $K$, the inequality (17) follows immediately from the inequality (16) and the holomorphicity of the entire function $F_{\alpha, 1}^{(\gamma)}(z)$ in the whole complex plane.

Theorem 4. Let $z \in \mathbb{C} ; n \in \mathbb{N}, \alpha>0, \gamma>0$. Then the Le Roy type functions $F_{\alpha, n}^{(\gamma)}$ satisfy the following asymptotic formula

$$
F_{\alpha, n}^{(\gamma)}(z)=\frac{1}{[\Gamma(n)]^{\gamma}}\left(1+\vartheta_{\alpha, n}^{(\gamma)}(z)\right), \quad \vartheta_{\alpha, n}^{(\gamma)}(z) \rightarrow 0 \text { as } n \rightarrow \infty .
$$

The functions $\vartheta_{\alpha, n}^{(\gamma)}(z)$ are holomorphic for $z \in \mathbb{C}$. The convergence is uniform in the nonempty compact subsets of the complex plane $\mathbb{C}$. 
Proof. The identities $(20)$ as well as the holomorphicity of $\vartheta_{\alpha, n}^{(\gamma)}(z)$ in the whole complex plane are obtained in Lemma 1. The rest follows immediately from the inequalities (16) and (17), Lemma 3.

Remark 5. According to the asymptotic formula (20), it follows that there exists a natural number $M$ such that the functions $[\Gamma(n)]^{\gamma} F_{\alpha, n}^{(\gamma)}(z)$ have not any zeros at all for $n>M$.

\section{Series in Le Roy type functions}

For simplicity of studying, we introduce auxiliary functions, related to the Le Roy functions, adding $\widetilde{F}_{\alpha, 0}^{(\gamma)}(z)$ just for completeness, namely:

$$
\widetilde{F}_{\alpha, 0}^{(\gamma)}(z)=1, \widetilde{F}_{\alpha, n}^{(\gamma)}(z)=z^{n}[\Gamma(n)]^{\gamma} F_{\alpha, n}^{(\gamma)}(z), n \in \mathbb{N} ; \alpha>0, \gamma>0,
$$

and we consider series in these functions:

$$
\sum_{n=0}^{\infty} a_{n} \widetilde{F}_{\alpha, n}^{(\gamma)}(z),
$$

for $z \in \mathbb{C}$ and with complex coefficients $a_{n}(n=0,1,2, \ldots)$.

Our main objective is to study the convergence of the series (22) in the complex plane. We propose results, corresponding to the classical CauchyHadamard theorem and Abel lemma for the power aeries. Such kind of results may be useful for studying the solutions of some fractional order differential and integral equations, expressed in terms of series (or series of integrals) in special functions of the type (21) (as for example in Kiryakova [16] in a more general case). Such kind of convergence theorems have been also obtained for series in other special functions, for example, for series in Laguerre and Hermite polynomials [31]- [33], and resp. by the author for series in Bessel and MittagLeffler type functions in the previous papers [19]-[22], [24]-[26] and the book $[23]$.

Let us denote by $D(0 ; R)$ the open disk with the radius $R$ and centred at the origin, and let the circle $C(0 ; R)$ be its boundary, i.e.

$$
D(0 ; R):|z|<R \quad \text { and } \quad C(0 ; R):|z|<R \quad(z \in \mathbb{C}) .
$$

In the beginning, we give a theorem of the Cauchy-Hadamard type for the series (22). 
Theorem 6 (of Cauchy-Hadamard type). Let $z \in \mathbb{C}, n \in \mathbb{N}, \alpha>0, \gamma>0$. Then the domain of convergence of the series (22) with complex coefficients $a_{n}$ is the disk $D(0 ; R)$ with a radius of convergence

$$
R=1 / \limsup _{n \rightarrow \infty}\left(\left|a_{n}\right|\right)^{1 / n} .
$$

The cases $R=0$ and $R=\infty$ are included in the general case.

Proof. Denoting for convenience

$$
u_{n}(z)=a_{n} \widetilde{F}_{\alpha, n}^{(\gamma)}(z), \quad b_{n}=\left|a_{n}\right|^{1 / n}, \quad \Lambda=\limsup _{n \rightarrow \infty} b_{n},
$$

and using the asymptotic formula (20), we get

$$
u_{n}(z)=a_{n} z^{n}\left(1+\vartheta_{\alpha, n}^{(\gamma)}(z)\right), \quad \vartheta_{\alpha, 0}^{(\gamma)}(z)=0 .
$$

Further the proof goes separately in the following three cases.

1. $\Lambda=0$. Then $\lim _{n \rightarrow \infty} b_{n}=\limsup _{n \rightarrow \infty} b_{n}=0$. Let us fix $z \neq 0$. Obviously, because of (20), there exists a number $N_{1}$ such that $\left|1+\vartheta_{\alpha, n}^{(\gamma)}(z)\right|<2$ and $2 b_{n}<$ $1 /|z|$ hold for every $n>N_{1}$, which is equivalent to $\left|u_{n}(z)\right|=b_{n}^{n}|z|^{n}\left|1+\vartheta_{\alpha, n}^{(\gamma)}(z)\right|<$ $2^{1-n}$. The absolute convergence of the series (22) follows immediately from this inequality.

2. $0<\Lambda<\infty$. First, let $z$ be in the domain $D(0 ; R)(z \in \mathbb{C})$, i.e. $|z| / R=\Lambda|z|<1$. Then $\limsup b_{n}|z|<1$. Therefore, it exists a number $q<1$ such that $\limsup _{n \rightarrow \infty} b_{n}|z| \stackrel{n \rightarrow \infty}{\leq}$, whence $b_{n}^{n}|z|^{n} \leq q^{n}$. By using the asymptotic formula (20) for the general member $u_{n}(z)$ of the series (22), we obtain $\left|u_{n}(z)\right|=$ $b_{n}^{n}|z|^{n}\left|1+\vartheta_{n}(z)\right| \leq q^{n}\left|1+\vartheta_{n}(z)\right|$. Since $\lim _{n \rightarrow \infty} \vartheta_{n}(z)=0$ there exists $N_{2}$ that $\left|1+\vartheta_{n}(z)\right|<2$ for every $n>N_{2}$, and hence $\left|u_{n}(z)\right| \leq 2 q^{n}$. Since the series $\sum_{n=0}^{\infty} 2 q^{n}$ is convergent, the series (22) is also convergent, even absolutely.

Now, let $z$ lie outside this domain. Then $|z| / R>1$ and $\lim \sup |z| b_{n}>1$. Therefore there exists infinite number of values $n_{k}$ of $n:|z|^{n} n_{k}^{n \rightarrow \infty} b_{n_{k}}^{n}>1$. Since $\lim _{n \rightarrow \infty} \vartheta_{n}(z)=0$, there exists $N_{3}$ so that $\left|1+\vartheta_{n_{k}}(z)\right| \geq 1 / 2$, for $n_{k}>N_{3}$, i.e. $\left|u_{n}(z)\right| \geq 1 / 2$ for an infinite number of values of $n$. That means that the necessary condition for convergence is not satisfied. Therefore the series (22) is divergent.

3. $\Lambda=\infty$. Let $z \in \mathbb{C} \backslash\{0\}$. Then $b_{n_{k}}>1 /|z|$ for an infinite number of values $n_{k}$ of $n$, and from here it follows $\left|u_{n_{k}}(z)\right|=|z|^{n_{k}} b_{n_{k}}^{n_{k}}\left|1+\vartheta_{n_{k}}(z)\right| \geq 1 / 2$ (again for $n_{k}>N_{3}$ ). That means that the necessary condition for the convergence of 
the series (22) is not satisfied and we conclude that the series (22) is divergent for every $z \neq 0$.

Thus, the considered series (22) absolutely converges in the disk $D(0 ; R)$ with the radius $R$, given by (23), like in the classical theory of the power series. Additionally, it turns out that the convergence of the discussed series is uniform inside the disk, i.e., the following corollary, similar to the classical Abel lemma, holds.

Corollary 7. Let $z \in \mathbb{C}, n \in \mathbb{N}, \alpha>0, \gamma>0$, and let the series (22) converge at the point $z_{0} \neq 0$. Then it is absolutely convergent in the disk $D\left(0 ;\left|z_{0}\right|\right)$. Inside the disk $D(0 ; R)$, i.e. on each closed disk $|z| \leq r<R(R$ defined by (23)), the convergence is uniform.

Proof. Indeed, since the considered series converges at the point $z_{0} \neq 0$, its radius of convergence is the positive number $R$, and moreover the point $z_{0}$ lies either in the disk $D(0 ; R)$ or on its boundary - the circle $C(0 ; R)$. That is why, the disk $D\left(0 ;\left|z_{0}\right|\right)$ is either a part of the region of convergence or coincide with it, whence the absolute convergence follows. To prove uniformity of the convergence inside the disk $D(0 ; R)$, it means to show that the series is uniformly convergent on each closed disk $|z| \leq r<R$. To this purpose, choosing a point $\zeta,|\zeta|=\rho, r<\rho<R$ and considering the series $(22)$, we estimate $\left|a_{n} \widetilde{F}_{\alpha, n}^{(\gamma)}(z)\right|$. First, mention that due to Remark 5 , some of the values of $\widetilde{F}_{\alpha, n}^{(\gamma)}(\zeta)$, but only a finite numbers of them, can be zero. Then there exists a number $P$ such that

$$
\left|a_{n} \widetilde{F}_{\alpha, n}^{(\gamma)}(z)\right|=\left|a_{n} \widetilde{F}_{\alpha, n}^{(\gamma)}(\zeta)\right| \frac{\left|\widetilde{F}_{\alpha, n}^{(\gamma)}(z)\right|}{\left|\widetilde{F}_{\alpha, n}^{(\gamma)}(\zeta)\right|} \leq\left|a_{n} \widetilde{F}_{\alpha, n}^{(\gamma)}(\zeta)\right| \frac{\left|1+\vartheta_{\alpha, n}^{(\gamma)}(z)\right|}{\left|1+\vartheta_{\alpha, n}^{(\gamma)}(\zeta)\right|}
$$

for all $n>P$ and $|z| \leq r$.

Because of inequality (17) and the $\Gamma$-functions quotient property it follows that

$$
\left|\vartheta_{\alpha, n}^{(\gamma)}(z)\right|=O\left(\frac{1}{n^{\alpha \gamma}}\right) .
$$

Then, due to the last relation and along with

$$
\lim _{n \rightarrow \infty} \frac{1}{n^{\alpha \gamma}}=0, \quad \lim _{n \rightarrow \infty}\left(1+\vartheta_{\alpha, n}^{(\gamma)}(\zeta)\right)^{-1}=1
$$

there exist numbers $A$ and $B$ such that $\left|1+\vartheta_{\alpha, n}^{(\gamma)}(z)\right|\left|1+\vartheta_{\alpha, n}^{(\gamma)}(\zeta)\right|^{-1} \leq A B$ and hence, $\left|a_{n} \widetilde{F}_{\alpha, n}^{(\gamma)}(z)\right| \leq A B\left|a_{n} \widetilde{F}_{\alpha, n}^{(\gamma)}(\zeta)\right|$, for all the values of $n>P$ and $|z| \leq r$. 
Since the series $\sum_{n=0}^{\infty} a_{n} \widetilde{F}_{\alpha, n}^{(\gamma)}(\zeta)$ is absolutely convergent and by the Weierstrass comparison criterium, the uniform convergence of the series (22) is proved.

So, the series (22) absolutely converges in the open disk $D(0 ; R)$ and diverges in its outside. Inside the disk $D(0 ; R)$, i.e. on each closed disk $|z| \leq r<R$ ( $R$ defined by (23)), the convergence is uniform. However, the very disk of convergence is not obligatorily a region of uniform convergence, and on its boundary the series may even be divergent. More precise results, connected with the behaviour of the series $(22)$ 'near' the boundary $C(0 ; R)$ as well as on it will be discussed and published elsewhere.

\section{Acknowledgements}

This paper is performed in the frames of the Bilateral Res. Projects 'Operators, differential equations and special functions of Fractional Calculus numerics and applications' between BAS and SANU and 'Analysis, Geometry and Topology' between BAS and MANU. It is also under the COST program, COST Action CA15225 'Fractional'.

\section{References}

[1] M.-A. Al-Bassam, Y.F. Luchko, On generalized fractional calculus and it application to the solution of integro-differential equations, J. Fract. Calc., 7 (1995), 69-88.

[2] R.W. Conway, W.L. Maxwell, A queueing model with state dependent service rate, J. Industr. Engn., 12, No 2 (1962), 132-136.

[3] M.M. Dzrbashjan, Integral Transforms and Representations in the Complex Domain (in Russian), Nauka, Moscow (1966).

[4] M.M. Dzrbashjan, Interpolation and Spectrum Expansions Associated with Fractional Differential Operators (in Russian), Erevan (1983).

[5] R. Garra, F. Polito, On some operators involving Hadamard derivatives, Integr. Trans. Spec. Func., 24, No 10 (2013), 773-782.

[6] R. Garrappa, S. Rogosin, F. Mainardi, On a generalized three-parameter Wright function of Le Roy type, Fract. Calc. Appl. Anal., 20, No 5 (2017), 1196-1215; DOI: 10.1515/fca-2017-0063. 
[7] S. Gerhold, Asymptotics for a variant of the Mittag-Leffler function, Integr. Trans. Spec. Func., 23, No 6 (2012), 397-403.

[8] R. Gorenflo, A.A. Kilbas, F. Mainardi, S.V. Rogosin, Mittag-Leffler Functions, Related Topics and Applications, Springer (2014); DOI: 10.1007/9783-662-43930-2; 2nd Ed. (2020); DOI: 10.1007/978-3-662-61550-8.

[9] R. Gorenflo, F. Mainardi, Fractional calculus: integral and differential equations of fractional order. In: A. Carpinteri and F. Mainardi (Eds.), Fractals and Fractional Calculus in Continuum Mechanics, SpringerVerlag, Wien (1997), 223-276.

[10] A.A. Kilbas, A.A. Koroleva, S.V. Rogosin, Multi-parametric Mittag-Leffler functions and their extension, Fract. Calc. Appl. Anal., 16, No 2 (2013), 378-404; DOI: 10.2478/s13540-013-0024-9.

[11] A.A. Kilbas, H.M. Srivastava, J.J. Trujillo, Theory and Applications of Fractional Differential Equations, Elsevier (2006).

[12] V. Kiryakova, Multiindex Mittag-Leffler functions, related GelfondLeontiev operators and Laplace type integral transforms, Fract. Calc. Appl. Anal., 2, No 4 (1999), 445-462.

[13] V. Kiryakova, Multiple (multiindex) Mittag-Leffler functions and relations to generalized fractional calculus, J. Comput. Appl. Math., 118 (2000), 241-259; doi: 10.1016/S0377-0427(00)00292-2.

[14] V. Kiryakova, The multi-index Mittag-Leffler functions as important class of special functions of fractional calculus, Computers and Math. with Appl., 59, No 5 (2010), 1885-1895; doi: 10.1016/j.camwa.2009.08.025.

[15] V. Kiryakova, The special functions of fractional calculus as generalized fractional calculus operators of some basic functions, Computers and Math. with Appl., 59, No 3 (2010), 1128-1141; doi: 10.1016/j.camwa.2009.05.014.

[16] V. Kiryakova, Fractional order differential and integral equations with Erdélyi-Kober operators: Explicit solutions by means of the transmutation method, AIP Conf. Proc., 1410 (2011), 247-258, doi:10.1063/1.3664376.

[17] É. Le Roy, Valéurs asymptotiques de certaines séries procédant suivant les puissances entères et positives d'une variable réelle (In French), Darboux Bull. (2), 24 (1899), 245-268. 
[18] F. Mainardi, R. Gorenflo, Time-fractional derivatives in relaxation processes: a tutorial survey, Fract. Calc. Appl. Anal., 10, No 3 (2007), 269308; at http://www.math.bas.bg/ fcaa.

[19] J. Paneva-Konovska, Theorems on the convergence of series in generalized Lommel-Wright functions. Fract. Calc. Appl. Anal., 10, No 1 (2007), 5974; at http://www.math.bas.bg/ fcaa.

[20] J. Paneva-Konovska, Cauchy-Hadamard, Abel and Tauber type theorems for series in generalized Bessel-Maitland functions, Compt. Rend. Acad. Bulg. Sci., 61, No 1 (2008), 9-14.

[21] J. Paneva-Konovska, Convergence of series in Mittag-Leffler functions, Compt. rend. Acad. bulg. Sci., 63, No 6 (2010), 815-822.

[22] J. Paneva-Konovska, A family of hyper-Bessel functions and convergent series in them, Fract. Calc. Appl. Anal., 17, No 4 (2014), 1001-1015; DOI: 10.2478/s13540-014-0211-3.

[23] J. Paneva-Konovska, From Bessel to Multi-Index Mittag Leffler Functions: Enumerable Families, Series in them and Convergence, World Scientific Publ., London (2016); doi: 10.1142/q0026.

[24] J. Paneva-Konovska, Periphery behaviour of series in Mittag-Leffler type functions, I, Intern. J. Appl. Math., 29, No 1 (2016), 69-78; doi: 10.12732/ijam.v29i1.6.

[25] J. Paneva-Konovska, Periphery behaviour of series in Mittag-Leffler type functions, II, Intern. J. Appl. Math., 29, No 2 (2016), 175-186; doi: 10.12732/ijam.v29i2.2.

[26] J. Paneva-Konovska, Overconvergence of series in generalized MittagLeffler functions, Fract. Calc. Appl. Anal., 20, No 2 (2017), 506-520; DOI: $10.1515 /$ fca-2017-0026.

[27] I. Podlubny, Fractional Differential Equations, Acad. Press (1999).

[28] T. Pogány, Integral form of the COM-Poisson renormalization constant, Statistics and Probability Letters, 119 (2016), 144-145.

[29] S. Rogosin, The role of the Mittag-Leffler function in fractional modeling, Mathematics, 3 (2015), 368-381. 
[30] M.F. Santarelli, D. Della Latta, M. Scipioni, V. Positano, L. Landini, A Conway-Maxwell-Poisson (CMP) model to address data dispersion on positron emission tomography, Computers in Biology and Medicine, 77 (2016), 90-101; doi: 10.1016/j.compbiomed.2016.08.006.

[31] P. Rusev, A theorem of Tauber type for the summation by means of Laguerre's polynomials, Compt. Rend. Acad. Bulg. Sci., 30, No 3 (1977), 331-334 (in Russian).

[32] P. Rusev, Analytic Functions and Classical Orthogonal Polynomials, Publ. House Bulg. Acad. Sci., Sofia (1984).

[33] P. Rusev, Classical Orthogonal Polynomials and Their Associated Functions in Complex Domain, Publ. House Bulg. Acad. Sci., Sofia (2005).

[34] S. Yakubovich and Yu. Luchko, The Hypergeometric Approach to Integral Transforms and Convolutions, Kluwer Acad. Publ., Dordrecht - Boston London (1994). 
